\title{
Gate-tunable cross-plane heat dissipation in single-layer transition metal dichalcogenides
}

\author{
Zhun-Yong Ong $\odot$, ' Gang Zhang, and Yong-Wei Zhang \\ Institute of High Performance Computing, A*STAR, Singapore 138632, Singapore \\ Linyou Cao \\ Department of Materials Science and Engineering, North Carolina State University, Raleigh, North Carolina 27695, USA; \\ Department of Physics, North Carolina State University, Raleigh, North Carolina 27695, USA; \\ and Department of Electrical and Computer Engineering, North Carolina State University, Raleigh, North Carolina 27695, USA
}

(Received 12 May 2020; revised 27 July 2020; accepted 25 August 2020; published 23 September 2020)

\begin{abstract}
Efficient heat dissipation to the substrate is crucial for optimal device performance in nanoelectronics. We develop a theory of electronic thermal boundary conductance (TBC) mediated by remote phonon scattering for the single-layer transition metal dichalcogenide (TMD) semiconductors $\mathrm{MoS}_{2}$ and $\mathrm{WS}_{2}$, and model their electronic TBC with different dielectric substrates $\left(\mathrm{SiO}_{2}, \mathrm{HfO}_{2}\right.$, and $\left.\mathrm{Al}_{2} \mathrm{O}_{3}\right)$. Our results indicate that the electronic TBC is strongly dependent on the electron density, suggesting that it can be modulated by the gate electrode in field-effect transistors, and this effect is most pronounced with $\mathrm{Al}_{2} \mathrm{O}_{3}$. Our work paves the way for the design of novel thermal devices with gate-tunable cross-plane heat-dissipative properties.
\end{abstract}

DOI: 10.1103/PhysRevResearch.2.033470

\section{INTRODUCTION}

Atomically thin two-dimensional (2D) transition metal dichalcogenide (TMD) semiconductors such as $\mathrm{MoS}_{2}$ and $\mathrm{WS}_{2}$ are promising alternative materials for the development of next-generation electronic devices [1,2]. At the nanoscale, the high power densities in these devices require efficient thermal management which is crucial for optimal device performance, with the thermal boundary conductance (TBC) of the $2 \mathrm{D}$ crystal-substrate interface playing a key role in the dissipation of excess Joule heat [3,4]. Therefore, clearer insights into the physical mechanisms underlying heat dissipation across the TMD-substrate interface may lead to the development of superior thermally aware TMD-based nanoelectronic designs, as well as novel applications in thermal energy harvesting or for channeling heat in ultracompact geometries.

One widely studied mechanism is the van der Waals coupling between the phonons of the 2D crystal and its substrate, which is believed to be the dominant component in the overall TBC [4]. The phononic TBC has been estimated using molecular dynamics (MD) simulations [5,6], elasticity theory [7-9], and density-functional-theory-based models [10]. Another mechanism of heat dissipation is the inelastic scattering of electrons in the 2D crystal by dipoles in the dielectric substrate, a phenomenon known widely as "remote phonon (RP)

\footnotetext{
*ongzy@ihpc.a-star.edu.sg

Published by the American Physical Society under the terms of the Creative Commons Attribution 4.0 International license. Further distribution of this work must maintain attribution to the author(s) and the published article's title, journal citation, and DOI.
}

scattering" or "surface optical phonon scattering" [11-14]. This phenomenon occurs for an insulating metal-oxide dielectric substrate (e.g., $\mathrm{SiO}_{2}$ ) in which the oscillating dipoles originating from the bulk polar optical phonons create an evanescent surface electric field that scatters the electrons remotely. The associated electron-phonon coupling strength depends on the dielectric properties of the substrate and screening by the electrons in the TMD. In a high- $\kappa$ dielectric substrate such as $\mathrm{HfO}_{2}$ or $\mathrm{Al}_{2} \mathrm{O}_{3}$, inelastic RP scattering can be strong enough to cause substantial momentum relaxation of the TMD electrons [15]. In graphene [14,16], this mechanism is also expected to facilitate energy dissipation from the nonequilibrium electrons to the substrate.

In spite of its role in limiting the electron mobility in TMDs $[15,17]$, our understanding of heat dissipation through RP scattering remains limited for TMDs. Unlike the phononic TBC which depends on interatomic forces, the RP-mediated electronic TBC $\left(G_{\mathrm{el}}\right)$ is expected to vary with the electron density $(n)$, which can be modulated by the gate electrode in a field-effect transistor. Thus, a deeper understanding of this phenomenon may inspire the design of novel devices with gate-tunable thermal properties and perhaps shed light on the distribution of reported experimental TBC values [18-21]. Although earlier theoretical and experimental work on RP heat dissipation in graphene [16,22] indicates that $G_{\mathrm{el}}$ is small for the graphene- $\mathrm{SiO}_{2}$ interface and weakly dependent on the electron density, the different electronic structure in singlelayer $\mathrm{MoS}_{2}$ and $\mathrm{WS}_{2}$ suggests that these findings for graphene may not apply to TMDs.

In our paper, we develop a theoretical model of heat dissipation by RP scattering [16] and apply it to investigate the electronic $\mathrm{TBC}$ and its dependence on the electron density, substrate material $\left(\mathrm{SiO}_{2}, \mathrm{HfO}_{2}\right.$, and $\left.\mathrm{Al}_{2} \mathrm{O}_{3}\right)$, and temperature $(T)$ in two commonly studied single-layer TMDs 
$\left(\mathrm{MoS}_{2}\right.$ and $\left.\mathrm{WS}_{2}\right)$. The effects of electron screening on $G_{\mathrm{el}}$ are studied. One of our main findings is that the $G_{\mathrm{el}}$ for $\mathrm{MoS}_{2}$ and $\mathrm{WS}_{2}$ exhibits a substantially greater dependence on $n$ than the $G_{\mathrm{el}}$ for graphene, with $G_{\mathrm{el}}$ reaching $85 \mathrm{MW} / \mathrm{K} / \mathrm{m}^{2}$ for the $\mathrm{MoS}_{2}-\mathrm{Al}_{2} \mathrm{O}_{3}$ interface at the electron density of $10^{13} \mathrm{~cm}^{-2}$. We suggest applications for different substrate materials and how this electron density dependence of the TBC can be exploited to create gate-tunable thermal insulators.

\section{THEORETICAL MODEL FOR HEAT DISSIPATION BY REMOTE PHONON SCATTERING}

The key elements of our RP model are based on Ref. [16]. We treat the electrons in the TMD as a single-band 2D electron gas (2DEG) at a fixed distance $d$ above the substrate, which we approximate as a dielectric continuum. The treatment of the TMD as a $2 \mathrm{D}$ electron gas can be justified by the three-atom thickness of single-layer TMD crystals, which implies that the electrons are strongly confined in the out-of-plane direction. The 2DEG in the TMD has a parabolic dispersion characterized by the effective mass $m_{e}$ with spin and valley degeneracies $g_{s}$ and $g_{v}$, respectively. For the substrate, the bulk polar optical phonons, of which there are typically two branches for a dielectric insulator such as $\mathrm{SiO}_{2}$, are characterized by their longitudinal optical (LO) and transverse optical (TO) frequencies [12], which are related through the equation $\epsilon_{\mathrm{sub}}^{0}=\epsilon_{\mathrm{sub}}^{\infty}\left(\frac{\omega_{\mathrm{LO1}}^{2}}{\omega_{\mathrm{TO} 1}^{2}}\right)\left(\frac{\omega_{\mathrm{LO} 2}^{2}}{\omega_{\mathrm{TO} 2}^{2}}\right)$, where $\epsilon_{\text {sub }}^{0}$ and $\epsilon_{\text {sub }}^{\infty}$ represent, respectively, the static and optical permittivity of the substrate, and $\omega_{\mathrm{LO} 1}$ and $\omega_{\mathrm{LO} 2}\left(\omega_{\mathrm{TO} 1}\right.$ and $\left.\omega_{\mathrm{TO} 2}\right)$ are, respectively, the LO (TO) frequencies of the first and second phonon branches with $\omega_{\mathrm{LO} 1}<\omega_{\mathrm{LO} 2}$ $\left(\omega_{\mathrm{TO} 1}<\omega_{\mathrm{TO} 2}\right)$. The LO phonon frequencies are determined from the roots of the frequency-dependent substrate dielectric function

$$
\begin{aligned}
\epsilon_{\mathrm{sub}}(\omega)= & \epsilon_{\mathrm{sub}}^{\infty}+\left(\epsilon_{\mathrm{sub}}^{i}-\epsilon_{\mathrm{sub}}^{\infty}\right) \frac{\omega_{\mathrm{TO} 2}^{2}}{\omega^{2}-\omega_{\mathrm{TO} 2}^{2}} \\
& +\left(\epsilon_{\mathrm{sub}}^{0}-\epsilon_{\mathrm{sub}}^{i}\right) \frac{\omega_{\mathrm{TO} 1}^{2}}{\omega^{2}-\omega_{\mathrm{TO} 1}^{2}},
\end{aligned}
$$

where $\omega$ and $\epsilon_{\mathrm{sub}}^{i}$ are, respectively, the frequency and intermediate permittivity of the substrate. We can rewrite Eq. (1) as $\epsilon_{\mathrm{sub}}(\omega)=\epsilon_{\mathrm{sub}}^{\infty}\left(\frac{\omega_{\mathrm{LO} 1}^{2}-\omega^{2}}{\omega_{\mathrm{TO} 1}^{2}-\omega^{2}}\right)\left(\frac{\omega_{\mathrm{LO} 2}^{2}-\omega^{2}}{\omega_{\mathrm{TO} 2}^{2}-\omega^{2}}\right)$. The corresponding surface optical (SO) frequencies associated with the surface electric field, $\omega_{\mathrm{SO} 1}$ and $\omega_{\mathrm{SO} 2}$, are determined from the solutions to $\epsilon_{\text {sub }}(\omega)+\epsilon_{0}=0$ [13]. The electronic TBC is determined from the rate of change of the SO1 and SO2 phonons as they scatter with the electrons in the TMD and the resultant rate of energy dissipation from the electrons, following the approach in Ref. [16].

\section{A. Surface optical phonon emission and absorption rates}

From first-order perturbation theory, the rate of change in the Bose-Einstein (BE) occupation factor $N_{\gamma, \boldsymbol{q}}$ of the $\gamma$ phonon with wave vector $q$, where $\gamma=$ SO1 and SO2 indexes the SO phonon branch, is [16]

$$
\frac{d N_{\gamma, \boldsymbol{q}}}{d t}=-g_{s} g_{v} \sum_{\boldsymbol{p}}\left(W_{\gamma, \boldsymbol{p} \rightarrow \boldsymbol{p}+\boldsymbol{q}}^{(\mathrm{abs})}-W_{\gamma, \boldsymbol{p} \rightarrow \boldsymbol{p}-\boldsymbol{q}}^{(\mathrm{ems})}\right) .
$$

The phonon absorption $\left(W_{\gamma, p \rightarrow p+q}^{(\mathrm{abs})}\right)$ and emission $\left(W_{\gamma, p \rightarrow p-q}^{(\mathrm{ems})}\right)$ terms describe phonon absorption and emission due to the $\boldsymbol{p} \rightarrow \boldsymbol{p}+\boldsymbol{q}$ and $\boldsymbol{p} \rightarrow \boldsymbol{p}-\boldsymbol{q}$ electronic transitions, respectively, where $\boldsymbol{p}$ is the wave vector of the initial electronic state. The expressions for $W_{\gamma, \boldsymbol{p} \rightarrow \boldsymbol{p}+\boldsymbol{q}}^{(\mathrm{abs})}$ and $W_{\gamma, \boldsymbol{p} \rightarrow \boldsymbol{p}-\boldsymbol{q}}^{(\mathrm{ems}}$ can be derived from the Fermi golden rule to yield

$$
\left\{\begin{array}{c}
W_{\gamma, \boldsymbol{p} \rightarrow \boldsymbol{p}+\boldsymbol{q}}^{(\mathrm{abs})} \\
W_{\gamma, \boldsymbol{p} \rightarrow \boldsymbol{p}-\boldsymbol{q}}^{(\mathrm{ems})}
\end{array}\right\}=\frac{2 \pi\left|M_{\gamma, \boldsymbol{q}}\right|^{2}}{\hbar}\left\{\begin{array}{c}
f_{\boldsymbol{p}}\left(1-f_{\boldsymbol{p}+\boldsymbol{q}}\right) N_{\gamma, \boldsymbol{q}} \delta\left(E_{\boldsymbol{p}+\boldsymbol{q}}-E_{\boldsymbol{p}}-\hbar \omega_{\gamma}\right) \\
f_{\boldsymbol{p}}\left(1-f_{\boldsymbol{p}-\boldsymbol{q}}\right)\left(N_{\gamma, \boldsymbol{q}}+1\right) \delta\left(E_{\boldsymbol{p}-\boldsymbol{q}}-E_{\boldsymbol{p}}+\hbar \omega_{\gamma}\right)
\end{array}\right\},
$$

where $M_{\gamma, \boldsymbol{q}}$ is the electron-phonon coupling coefficient, $f_{p}=\left\{\exp \left[\beta\left(E_{p}-\mu\right)\right]+1\right\}^{-1}$ is the Fermi-Dirac occupation factor for the electronic state $\boldsymbol{p}, N_{\gamma, \boldsymbol{q}}$ is the BoseEinstein occupation factor $N\left(\omega_{\gamma}, T\right)=\left[\exp \left(\beta \hbar \omega_{\gamma}\right)-1\right]^{-1}$, and $E_{p}=\hbar^{2} p^{2} /\left(2 m_{e}\right)$ and $\omega_{\gamma}$ represent the electron and phonon energy, respectively. The terms $\beta=\left(k_{B} T\right)^{-1}$ and $\mu=$ $\beta^{-1} \ln \left[\exp \left(\frac{2 \pi \hbar^{2} n \beta}{g_{s} g_{v} m_{e}}\right)-1\right]$ represent, respectively, the inverse temperature and chemical potential, where $T$ is the temperature, $n$ is the electron density, $k_{B}$ is the Boltzmann constant, and $\hbar$ is the reduced Planck constant.

Given Eq. (3), the sums in Eq. (2) can be written as

$\sum_{\boldsymbol{p}}\left\{\begin{array}{l}W_{\gamma, \boldsymbol{p} \rightarrow \boldsymbol{p}+\boldsymbol{q}}^{(\mathrm{abs})} \\ W_{\gamma, \boldsymbol{p} \rightarrow \boldsymbol{p}-\boldsymbol{q}}^{(\mathrm{ems})}\end{array}\right\}=\frac{2 \pi \Omega\left|M_{\gamma, \boldsymbol{q}}\right|^{2}}{g_{s} g_{v} \hbar}\left\{\begin{array}{c}N_{\gamma, \boldsymbol{q}} S_{0}\left(\boldsymbol{q}, \omega_{\gamma}\right) \\ \left(N_{\gamma, \boldsymbol{q}}+1\right) S_{0}\left(-\boldsymbol{q},-\omega_{\gamma}\right)\end{array}\right\}$,

where $\Omega$ is the area of the TMD-substrate interface and $S_{0}(\boldsymbol{q}, \omega)$ is the dynamic structure factor of the 2DEG in the random phase approximation [23], i.e., $S_{0}(\boldsymbol{q}, \omega)=$

$$
\begin{gathered}
\frac{g_{s} g_{v}}{\Omega} \sum_{p} f_{\boldsymbol{p}}\left(1-f_{\boldsymbol{p}+\boldsymbol{q}}\right) \delta\left(E_{\boldsymbol{p}+\boldsymbol{q}}-E_{\boldsymbol{p}}-\hbar \omega\right), \text { which simplifies to } \\
S_{0}(\boldsymbol{q}, \omega)=\frac{g_{s} g_{v}[N(\omega, T)+1]}{\Omega \pi} \lim _{\eta \rightarrow 0^{+}} \\
\quad \times \operatorname{Im} \sum_{\boldsymbol{p}} \frac{f_{\boldsymbol{p}+\boldsymbol{q}}-f_{\boldsymbol{p}}}{E_{\boldsymbol{p}+\boldsymbol{q}}-E_{\boldsymbol{p}}-\hbar \omega+i \eta} \\
=\frac{N(\omega, T)+1}{\pi} \operatorname{Im} \mathcal{P}(\boldsymbol{q}, \omega ; \mu, T)
\end{gathered}
$$

and

$$
\mathcal{P}(\boldsymbol{q}, \omega ; \mu, T)=\frac{g_{s} g_{v}}{\Omega} \lim _{\eta \rightarrow 0^{+}} \sum_{\boldsymbol{p}} \frac{f_{\boldsymbol{p}+\boldsymbol{q}}-f_{\boldsymbol{p}}}{\hbar \omega-E_{\boldsymbol{p}+\boldsymbol{q}}+E_{\boldsymbol{p}}+i \eta} .
$$

Equation (4) describes the finite-temperature 2DEG polarizability and can be written as [24]

$$
\mathcal{P}\left(\boldsymbol{q}, \omega ; \mu, T_{\mathrm{el}}\right)=\int_{0}^{\infty} d \mu^{\prime} \frac{\mathcal{P}\left(\boldsymbol{q}, \omega ; \mu^{\prime}, 0\right)}{4 k_{B} T_{\mathrm{el}} \cosh ^{2}\left(\frac{\mu-\mu^{\prime}}{2 k_{B} T_{\mathrm{el}}}\right)},
$$


where $T_{\mathrm{el}}$ is the electronic temperature and, for $z_{ \pm}=\hbar \omega \pm \frac{\hbar^{2} q^{2}}{2 m_{e}}$ and $u=\frac{2 \mu \hbar^{2} q^{2}}{m_{e}}$, its exact expression at $T_{\mathrm{el}}=0$ is [24]

$$
\begin{aligned}
\mathcal{P}(\boldsymbol{q}, \omega ; \mu, 0)= & \frac{g_{s} g_{v} m_{e}}{2 \pi \hbar^{2}}+\frac{g_{s} g_{v} m_{e}^{2}}{2 \pi \hbar^{4} q^{2}}\left\{\operatorname{sgn}\left(z_{+}\right) \Theta\left(z_{+}^{2}-u\right) \sqrt{z_{+}^{2}-u}-\operatorname{sgn}\left(z_{-}\right) \Theta\left(z_{-}^{2}-u\right) \sqrt{z_{-}^{2}-u}\right\} \\
& +i \frac{g_{s} g_{v} m_{e}^{2}}{2 \pi \hbar^{4} q^{2}}\left\{\Theta\left(u-z_{+}^{2}\right) \sqrt{u-z_{+}^{2}}-\Theta\left(u-z_{-}^{2}\right) \sqrt{u-z_{-}^{2}}\right\} .
\end{aligned}
$$

\section{B. Electron-phonon interaction and screening}

The electron-phonon coupling coefficient $M_{\gamma, q}$ in Eq. (3) is [13]

$$
M_{\gamma, q}=\left[\frac{e^{2} \hbar \omega_{\gamma} \exp (-2 q d)}{\Omega q \varepsilon(q)}\left(\frac{1}{\epsilon_{\gamma, \mathrm{hi}}}-\frac{1}{\epsilon_{\gamma, \mathrm{lo}}}\right)\right]^{1 / 2},
$$

where $e$ is the electron charge. The expressions for $\epsilon_{\mathrm{SO} 1 \text {,hi }}$, $\epsilon_{\mathrm{SO} 1, \mathrm{lo}}, \epsilon_{\mathrm{SO} 2, \mathrm{hi}}$, and $\epsilon_{\mathrm{SO} 2 \text {,lo }}$ in Eq. (5) are

$$
\begin{aligned}
\epsilon_{\mathrm{SO} 1, \mathrm{hi}} & =\frac{1}{2}\left[\epsilon_{\mathrm{sub}}^{\infty}\left(\frac{\omega_{\mathrm{LO} 2}^{2}-\omega_{\mathrm{SO} 1}^{2}}{\omega_{\mathrm{TO} 2}^{2}-\omega_{\mathrm{SO} 1}^{2}}\right)+\epsilon_{0}\right], \\
\epsilon_{\mathrm{SO} 1, \mathrm{lo}} & =\frac{1}{2}\left[\epsilon_{\mathrm{sub}}^{\infty}\left(\frac{\omega_{\mathrm{LO} 1}^{2}}{\omega_{\mathrm{TO} 1}^{2}}\right)\left(\frac{\omega_{\mathrm{LO} 2}^{2}-\omega_{\mathrm{SO} 1}^{2}}{\omega_{\mathrm{TO} 2}^{2}-\omega_{\mathrm{SO} 1}^{2}}\right)+\epsilon_{0}\right], \\
\epsilon_{\mathrm{SO} 2, \mathrm{hi}} & =\frac{1}{2}\left[\epsilon_{\mathrm{sub}}^{\infty}\left(\frac{\omega_{\mathrm{LO} 1}^{2}-\omega_{\mathrm{SO} 2}^{2}}{\omega_{\mathrm{TO} 1}^{2}-\omega_{\mathrm{SO} 2}^{2}}\right)+\epsilon_{0}\right], \\
\epsilon_{\mathrm{SO} 2, \mathrm{lo}} & =\frac{1}{2}\left[\epsilon_{\mathrm{sub}}^{\infty}\left(\frac{\omega_{\mathrm{LO} 2}^{2}}{\omega_{\mathrm{TO} 2}^{2}}\right)\left(\frac{\omega_{\mathrm{LO} 1}^{2}-\omega_{\mathrm{SO} 2}^{2}}{\omega_{\mathrm{TO} 1}^{2}-\omega_{\mathrm{SO} 2}^{2}}\right)+\epsilon_{0}\right],
\end{aligned}
$$

where $\epsilon_{0}$ is the permittivity of the vacuum, and the screening function $\varepsilon(q)$ is given by [25]

$\varepsilon(q)^{-1}=1+\frac{e^{2} \operatorname{Re} \mathcal{P}(\boldsymbol{q}, 0 ; \mu, T)}{2 \epsilon_{0} q}\left[1-\frac{\epsilon_{\mathrm{sub}}^{\infty}-\epsilon_{0}}{\epsilon_{\mathrm{sub}}^{\infty}+\epsilon_{0}} \exp (-2 q d)\right]$.

As expected, Eq. (7) becomes $\varepsilon(q)^{-1}=1+$ $e^{2} \operatorname{Re} \mathcal{P}(\boldsymbol{q}, 0 ; \mu, T) /\left(2 \epsilon_{0} q\right)$ in the $d \rightarrow \infty$ (i.e., no substrate) limit and $\varepsilon(q)^{-1}=1+e^{2} \operatorname{Re} \mathcal{P}(\boldsymbol{q}, 0 ; \mu, T) /\left[\left(\epsilon_{\text {sub }}^{\infty}+\epsilon_{0}\right) q\right]$ in the $d=0$ limit (i.e., no gap between the TMD and substrate). The screening function reduces the bare electron-phonon strength especially in the short wavelength $(q \rightarrow \infty)$ limit and originates from the polarization of the mobile electrons in the 2DEG to screen the surface electric field generated by the substrate. To obtain the bare electron-phonon strength, we can set $\varepsilon(q)^{-1}=1$.

\section{Electronic thermal boundary conductance}

Using the identities $S_{0}(\boldsymbol{q}, \omega)=e^{\beta \hbar \omega} S_{0}(\boldsymbol{q},-\omega)$ and $S_{0}(\boldsymbol{q}, \omega)=S_{0}(-\boldsymbol{q}, \omega)$, Eq. (2) simplifies to

$$
\frac{d N_{\gamma, \boldsymbol{q}}}{d t}=-\frac{2 \pi \Omega\left|M_{\gamma, \boldsymbol{q}}\right|^{2}}{\hbar} S_{0}\left(\boldsymbol{q}, \omega_{\gamma}\right) \frac{N_{\gamma, \boldsymbol{q}}\left(T_{\mathrm{sub}}\right)-N_{\gamma, \boldsymbol{q}}^{\mathrm{Eq}}}{N_{\gamma, \boldsymbol{q}}^{\mathrm{Eq}}+1},
$$

where $N_{\gamma, \boldsymbol{q}}^{\mathrm{Eq}}$ is the Bose-Einstein occupation factor at the TMD temperature. Given the SO phonon energy density $\varrho\left(T_{\text {sub }}\right)=$ $\frac{1}{\Omega} \sum_{\gamma, \boldsymbol{q}} \hbar \omega_{\gamma} N_{\gamma, \boldsymbol{q}}$, we can write the electronic TBC as the derivative of the rate of change of $\varrho\left(T_{\text {sub }}\right)$ with respect to $T_{\text {sub }}$, i.e.,

$$
\begin{aligned}
G_{\mathrm{el}} & =-\frac{d}{d T_{\mathrm{sub}}}\left[\frac{d \varrho\left(T_{\mathrm{sub}}\right)}{d t}\right]_{T_{\mathrm{sub}}=T} \\
& =\sum_{\gamma, \boldsymbol{q}} \frac{2 \pi \hbar \omega_{\gamma}^{2}}{k_{B} T^{2}}\left|M_{\gamma, \boldsymbol{q}}\right|^{2} S_{0}\left(\boldsymbol{q}, \omega_{\gamma}\right) N_{\gamma, \boldsymbol{q}}^{\mathrm{Eq}} .
\end{aligned}
$$

Hence, the final expression for $G_{\mathrm{el}}$ is

$$
G_{\mathrm{el}}=\sum_{\gamma, \boldsymbol{q}} \frac{2 \hbar \omega_{\gamma}^{2}}{k_{B} T^{2}}\left|M_{\gamma, \boldsymbol{q}}\right|^{2}\left(N_{\gamma, \boldsymbol{q}}^{\mathrm{Eq}}+1\right) N_{\gamma, \boldsymbol{q}}^{\mathrm{Eq}} \operatorname{Im} \mathcal{P}\left(\boldsymbol{q}, \omega_{\gamma} ; \mu, T\right),
$$

which, for the ease of numerical evaluation, we can rewrite as a multivariable integral,

$$
\begin{aligned}
G_{\mathrm{el}}= & \sum_{\gamma=\mathrm{SO} 1, \mathrm{SO} 2} \mathcal{F}_{\gamma} \int_{0}^{q_{\max }} d q \frac{\exp (-2 q d)}{\varepsilon(q)} \\
& \times \int_{0}^{\infty} d \mu^{\prime} \frac{\operatorname{Im} \mathcal{P}\left(\boldsymbol{q}, \omega_{\gamma} ; \mu^{\prime}, 0\right)}{4 k_{B} T \cosh ^{2}\left(\frac{\mu-\mu^{\prime}}{2 k_{B} T}\right)}
\end{aligned}
$$

where $q_{\max }$ is the maximum wave vector, which we can set as $q_{\max }=2 \pi / \sqrt{A} \approx 2 \times 10^{10} \mathrm{~m}^{-1}$ ( $A$ is the unit cell area of the TMD), and

$$
\mathcal{F}_{\gamma}=\frac{e^{2} \hbar^{2} \omega_{\gamma}^{3}}{\pi k_{B} T^{2}}\left(N_{\gamma}^{\mathrm{Eq}}+1\right) N_{\gamma}^{\mathrm{Eq}}\left(\frac{1}{\epsilon_{\gamma, \mathrm{hi}}}-\frac{1}{\epsilon_{\gamma, \mathrm{lo}}}\right) .
$$

We use the simulation parameters from Table I in our calculations. The TMD-substrate gap size $d$ is a parameter in

TABLE I. Remote phonon scattering simulation parameters for $\mathrm{WS}_{2}$ and $\mathrm{MoS}_{2}$ [26]. The effective electron masses are expressed in terms of the free-electron mass $m_{0}$ and taken from Ref. [26]. The parameters $\epsilon_{\mathrm{sub}}^{\infty}, \epsilon_{\mathrm{sub}}^{i}, \epsilon_{\mathrm{sub}}^{0}, \omega_{\mathrm{TO} 1}$, and $\omega_{\mathrm{TO} 2}$ are taken from Ref. [27].

\begin{tabular}{lrcr}
\hline \hline Substrate & $\mathrm{SiO}_{2}$ & $\mathrm{Al}_{2} \mathrm{O}_{3}$ & $\mathrm{HfO}_{2}$ \\
\hline$m_{e} / m_{0}$ & \multicolumn{3}{c}{$0.31\left(\mathrm{WS}_{2}\right)$ and $0.51\left(\mathrm{MoS}_{2}\right)$} \\
$g_{s}$ & \multicolumn{3}{c}{$2\left(\mathrm{WS}_{2}\right.$ and $\left.\mathrm{MoS}_{2}\right)$} \\
$g_{v}$ & $2\left(\mathrm{WS}_{2}\right.$ and $\left.\mathrm{MoS}_{2}\right)$ & \\
$d(\mathrm{~nm})$ & 2.50 & 3.20 & 5.03 \\
$\epsilon_{\text {sub }}^{\infty} / \epsilon_{0}$ & 3.05 & 7.27 & 6.58 \\
$\epsilon_{\text {sub }}^{i} / \epsilon_{0}$ & 3.90 & 12.53 & 22.00 \\
$\epsilon_{\text {sub }}^{0} / \epsilon_{0}$ & 55.60 & 48.18 & 12.40 \\
$\omega_{\mathrm{TO} 1}(\mathrm{meV})$ & 138.10 & 71.41 & 48.35 \\
$\omega_{\mathrm{TO} 2}(\mathrm{meV})$ & 60.99 & 56.08 & 21.26 \\
$\omega_{\mathrm{SO} 1}(\mathrm{meV})$ & 148.97 & 110.11 & 55.08 \\
$\omega_{\mathrm{SO} 2}(\mathrm{meV})$ & & & \\
\hline \hline
\end{tabular}


a continuum model in which we treat the substrate as a continuous dielectric solid with a well-defined surface at which the dielectric function changes discontinuously from $\epsilon_{\text {sub }}(\omega)$ to $\epsilon_{0}$. However, there is no well-defined, exact point in atomistic models where we can say that the dielectric function changes suddenly. Hence, the precise value of $d$ cannot be determined from atomistic models. Moreover, the value of $d$ estimated from atomistic models can vary with the chemical configuration of the substrate surface. Thus, given the uncertainty in $d$, we look at the range of $G_{\mathrm{el}}$ values for different values of $d$, instead of computing $G_{\mathrm{el}}$ for a single fixed $d$ value.

We estimate $d=0.3 \mathrm{~nm}$ for the TMD-SiO 2 interfaces from the position of the highest substrate atom to the lowest TMD atom in the $\mathrm{TMD}-\mathrm{SiO}_{2}$ supercells optimized with density functional theory (DFT) calculations, the details of which are given in the Appendix. We also assume $d=0.3 \mathrm{~nm}$ for the TMD-HfO $\mathrm{O}_{2}$ and TMD- $\mathrm{Al}_{2} \mathrm{O}_{3}$ interfaces, for convenience. Our calculations are repeated for $d=0$ and $d=0.6 \mathrm{~nm}$ because of the strong $d$ dependence from the $e^{-2 q d}$ term in Eq. (9), to set the upper and lower bounds for $G_{\mathrm{el}}$, respectively.

\section{NUMERICAL RESULTS AND DISCUSSION}

To understand the experimental implications of the physics underlying the electronic TBC, we compute the electronic TBC $G_{\text {el }}$ using Eq. (9) for the single-layer TMDs $\left(\mathrm{MoS}_{2}\right.$ and $\left.\mathrm{WS}_{2}\right)$ and dielectric substrates $\left(\mathrm{SiO}_{2}, \mathrm{Al}_{2} \mathrm{O}_{3}\right.$, and $\left.\mathrm{HfO}_{2}\right)$ at different temperatures $(T)$ and electron densities $(n)$ since $T$ and $n$ can be varied in experiments. In field-effect transistors, the electron density is modulated by the gate electrode and can reach up to $n \sim 10^{13} \mathrm{~cm}^{-2}[15,17]$. Intuitively, we expect $G_{\mathrm{el}}$ to increase with $n$ as more electron-phonon scattering events occur at higher $n$. However, at higher $n$, the mobile electrons can also be polarized by an external electric field and generate a polarization field that attenuates the original external field. This effect is known as screening and it is expected to weaken the effective electron-phonon interaction, possibly offsetting the gain in $G_{\mathrm{el}}$ from the greater frequency of electron-phonon scattering. By studying the $n$ dependence of $G_{\text {el }}$, we clarify these two competing effects on $G_{\mathrm{el}}$.

\section{A. $G_{\text {el }}$ dependence on electron screening}

It is shown in Ref. [16] that $G_{\text {el }}$ in single-layer graphene can vary substantially depending on the form of the electronphonon interaction $M_{\gamma, q}$ in Eq. (5), with $G_{\mathrm{el}}$ for the bare or unscreened electron-phonon interaction (i.e., the unscreened $\left.G_{\mathrm{el}}\right)$ up to two orders of magnitude larger than $G_{\mathrm{el}}$ for the weaker screened electron-phonon interaction (i.e., the screened $\left.G_{\mathrm{el}}\right)$. Experimental TBC data from Koh and coworkers for the graphene- $\mathrm{SiO}_{2}$ interface [22] suggest that changes in the TBC are only weakly dependent on the electron density $n$, consistent with the screened $M_{\gamma, q}$. On the other hand, theories involving unscreened remote phonon interaction have been used to model heat dissipation in carbon nanotubes [28]. To settle this question for TMDs and to determine the significance of screening for $G_{\mathrm{el}}$ in TMDs, we compute the unscreened and screened $G_{\text {el }}$ using Eq. (9). For screened interactions, we use Eq. (7), while for unscreened interactions, we set $\varepsilon(q)^{-1}=1$ in Eq. (9).

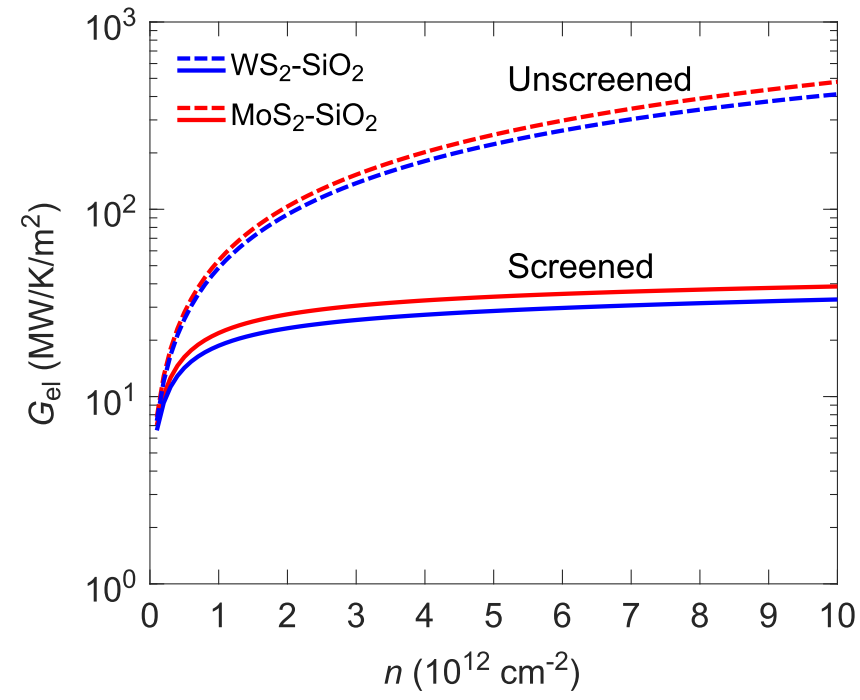

FIG. 1. Electron density dependence of $G_{\text {el }}$ for the $\mathrm{WS}_{2}-\mathrm{SiO}_{2}$ (blue lines) and $\mathrm{MoS}_{2}-\mathrm{SiO}_{2}$ (red lines) interfaces with screened (solid lines) and unscreened (dashed lines) interaction at $T=300 \mathrm{~K}$.

Figure 1 shows $G_{\mathrm{el}}$ as a function of $n$ at $T=300 \mathrm{~K}$ for the $\mathrm{MoS}_{2}-\mathrm{SiO}_{2}$ and $\mathrm{WS}_{2}-\mathrm{SiO}_{2}$ interfaces. For the $\mathrm{MoS}_{2}-\mathrm{SiO}_{2}$ interface, the unscreened $G_{\mathrm{el}}$ shows an order of magnitude rise from 53 to $479 \mathrm{MW} / \mathrm{K} / \mathrm{m}^{2}$ as $n$ increases from $10^{12}$ to $10^{13} \mathrm{~cm}^{-2}$, while the screened $G_{\text {el }}$ increases from 22 to $39 \mathrm{MW} / \mathrm{K} / \mathrm{m}^{2}$. This difference in the dependence on $n$ illustrates the significance of screening for the TBC. At low $n$ where screening is weak, the unscreened and screened $G_{\mathrm{el}}$ values are close, but at high $n$, the screening of $M_{\gamma, q}$ becomes more significant and the screened $G_{\mathrm{el}}$ diverges from the unscreened $G_{\mathrm{el}}$. We surmise that the large unscreened $G_{\text {el }}$ increase is unlikely to be correct given the much smaller $\mathrm{MoS}_{2}-\mathrm{SiO}_{2}$ TBC observed in Refs. [19,20]. Thus, electron screening must be taken into consideration in order to model electronic heat transfer at TMD-substrate interfaces. Nonetheless, even with the effects of screening, the $n$ dependence of $G_{\text {el }}$ is still significant and should be detectable in a $\mathrm{MoS}_{2}$ or $\mathrm{WS}_{2}$ field-effect transistor on a typical $\mathrm{Si} / \mathrm{SiO}_{2}$ substrate.

We observe that $G_{\text {el }}$ is larger for $\mathrm{MoS}_{2}$ than for $\mathrm{WS}_{2}$. At $n=10^{12} \mathrm{~cm}^{-2}$, the unscreened $G_{\mathrm{el}}$ is 53 and $49 \mathrm{MW} / \mathrm{K} / \mathrm{m}^{2}$ for the $\mathrm{MoS}_{2}-\mathrm{SiO}_{2}$ and $\mathrm{WS}_{2}-\mathrm{SiO}_{2}$ interface, respectively [16], i.e., $G_{\mathrm{el}}$ is $\sim 10$ percent higher for $\mathrm{MoS}_{2}$. This is due to the higher electron density of states for $\mathrm{MoS}_{2}$, which is a constant proportional to the electron effective mass $m_{e}$ [29] and corresponds to a greater amount of scattering. The relative difference is further enhanced for the screened $G_{\mathrm{el}}$, with the screened $G_{\mathrm{el}}$ for $\mathrm{MoS}_{2}$ about $\sim 17 \%$ higher, indicating that a higher $m_{e}$ also leads to weaker screening of the electronphonon interaction. This is because at a higher density of states, the greater availability of states lowers the chemical potential, which in turn decreases the screening strength. This combined effect of a lower density of states and stronger screening also explains why $G_{\mathrm{el}}$ is significantly lower for graphene. At $n=10^{12} \mathrm{~cm}^{-2}$, the unscreened $G_{\mathrm{el}}$ is 53 and $15 \mathrm{MW} / \mathrm{K} / \mathrm{m}^{2}$ for the $\mathrm{MoS}_{2}-\mathrm{SiO}_{2}$ and graphene-SiO 2 interface, respectively [16]. Unlike the 2DEG in $\mathrm{MoS}_{2}$ or $\mathrm{WS}_{2}$, which has a parabolic dispersion $\left(E \propto k^{2}\right)$, the $2 \mathrm{DEG}$ in 

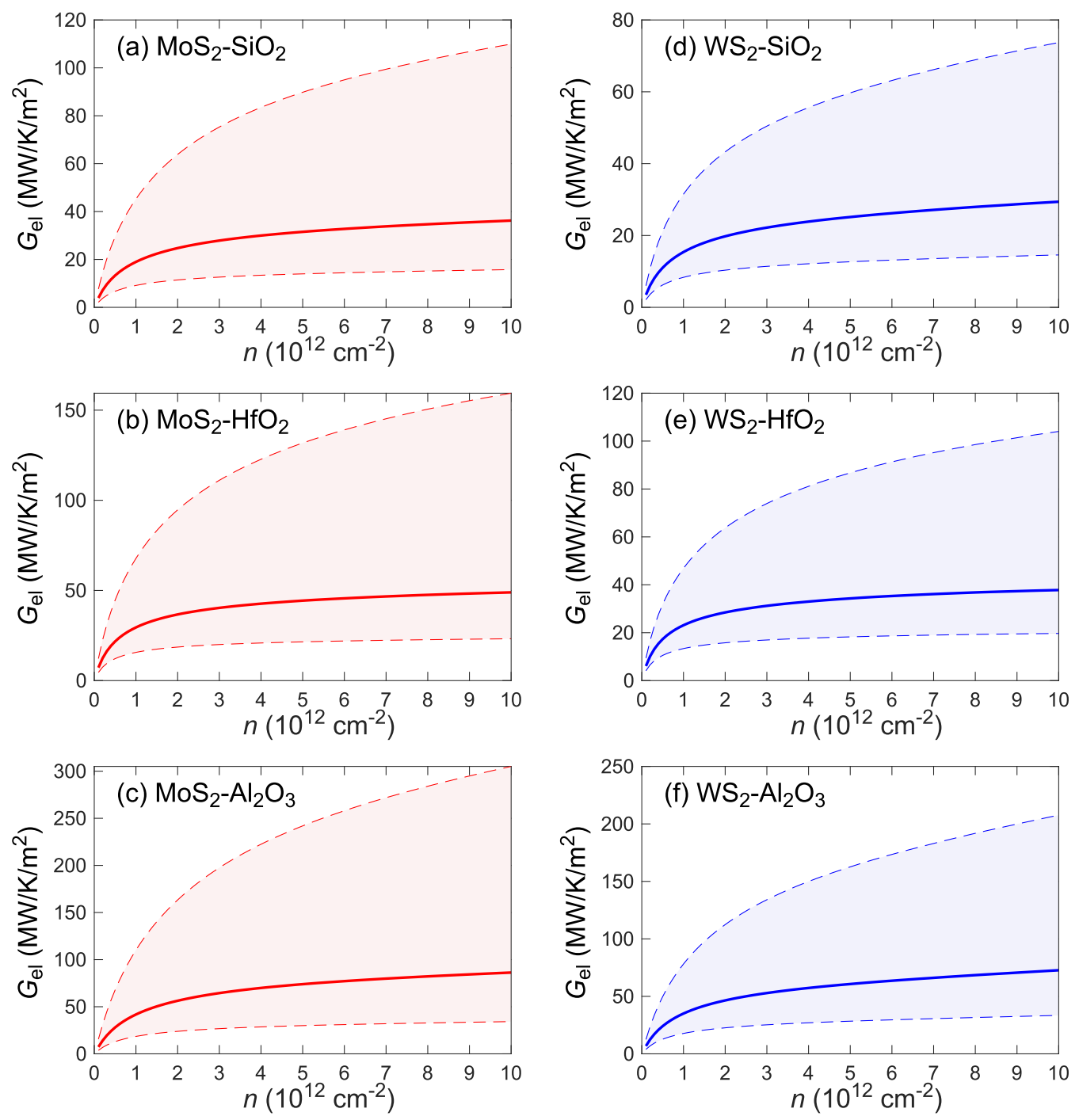

FIG. 2. Electron density dependence of $G_{\text {el }}$ for the TMD-substrate interface with different TMDs $\left(\mathrm{WS}_{2}\right.$ and $\left.\mathrm{MoS}_{2}\right)$ and substrates $\left(\mathrm{SiO}_{2}\right.$, $\mathrm{HfO}_{2}$, and $\mathrm{Al}_{2} \mathrm{O}_{3}$ ) at $T=300 \mathrm{~K}$ over the range of $n=0.1$ to $10 \times 10^{12} \mathrm{~cm}^{-2}$ for $d=0.3 \mathrm{~nm}$ (solid line). The upper and lower bounds in each panel correspond to $G_{\mathrm{el}}$ for $d=0$ and $d=0.6 \mathrm{~nm}$ (dashed lines).

graphene has a linear dispersion $(E \propto k)$ akin to a massless Dirac particle. Thus, the electronic density of states in graphene scales as $E$ near the Dirac point, which results in a reduced amount of scattering and stronger screening of the electron-phonon coupling, leading to a smaller $G_{\mathrm{el}}$.

\section{B. $G_{\mathrm{el}}$ dependence on substrate dielectric material, electron density, and temperature}

Figures 2(a) to 2(f) show the electronic TBC from Eq. (9) as a function of the electron density $n$ at $T=300 \mathrm{~K}$ for different substrates $\left(\mathrm{SiO}_{2}, \mathrm{HfO}_{2}\right.$, and $\left.\mathrm{Al}_{2} \mathrm{O}_{3}\right)$ and a TMD-substrate distance of $d=0.3 \mathrm{~nm}$. The lower and upper bounds for $G_{\mathrm{el}}$, corresponding to $d=0.6$ and $0.0 \mathrm{~nm}$, respectively, are also shown. As noted earlier, $G_{\mathrm{el}}$ increases monotonically from 0 with $n$ because of the higher rate of inelastic scattering events at higher $n$. However, the rate of increase of $G_{\text {el }}$ with respect to $n$ decreases at higher electron densities because the higher rate of inelastic scattering events is offset partially by the greater electron screening which weakens the electron-phonon coupling. We find that $G_{\mathrm{el}}$ is higher for $\mathrm{MoS}_{2}$ than $\mathrm{WS}_{2}$ with every substrate, and that $\mathrm{Al}_{2} \mathrm{O}_{3}$ is the substrate with the highest $G_{\mathrm{el}}$ because it has the highest bare electron-phonon coupling strength which we can characterize by the dimensionless parameter $\mathcal{C}=\epsilon_{0} / \epsilon_{\text {sub }}^{0}-\epsilon_{0} / \epsilon_{\text {sub }}^{\infty}(\mathcal{C}=0.147,0.153$, and 0.233 for $\mathrm{SiO}_{2}, \mathrm{HfO}_{2}$, and $\mathrm{Al}_{2} \mathrm{O}_{3}$, respectively). The higher $G_{\text {el }}$ for $\mathrm{MoS}_{2}$ can be explained by its greater electron effective mass which is proportional to the electron density of states [29].

By increasing the electron density to $n=10^{13} \mathrm{~cm}^{-2}$, which can be attained in $\mathrm{MoS}_{2}$ field-effect transistors [15,17], $G_{\text {el }}$ can reach a maximum of 85,48 , and $39 \mathrm{MW} / \mathrm{K} / \mathrm{m}^{2}$ in $\mathrm{Al}_{2} \mathrm{O}_{3}$, $\mathrm{HfO}_{2}$, and $\mathrm{SiO}_{2}$, respectively. This figure represents the gatetunable component of the overall TBC, which is the sum of $G_{\mathrm{el}}$ and $G_{\mathrm{ph}}$, the phononic TBC component, and suggests that the gate-tunable $G_{\text {el }}$ is most pronounced and easily observed for the TMD- $\mathrm{Al}_{2} \mathrm{O}_{3}$ interface at room temperature since $G_{\mathrm{el}} \gg G_{\mathrm{ph}}$, as theoretical results from Ref. [30] estimate that $G_{\mathrm{ph}}<4 \mathrm{MW} / \mathrm{K} / \mathrm{m}^{2}$ for the TMD- $\mathrm{Al}_{2} \mathrm{O}_{3}$ interface. This 

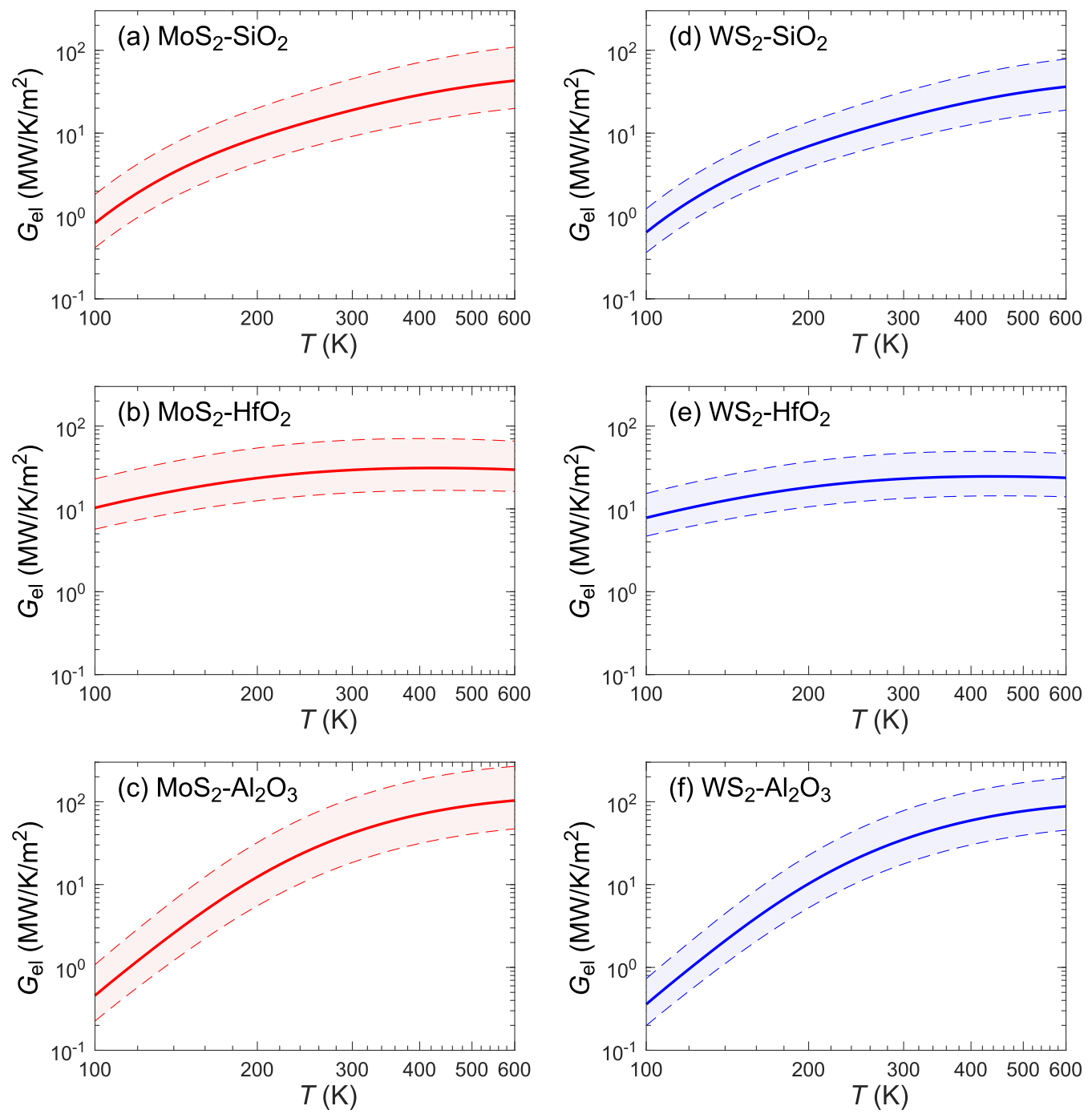

FIG. 3. Temperature dependence of $G_{\text {el }}$ for the TMD-substrate interface with different TMDs $\left(\mathrm{WS}_{2}\right.$ and $\left.\mathrm{MoS}_{2}\right)$ and substrates $\left(\mathrm{SiO}_{2}, \mathrm{HfO}_{2}\right.$, and $\mathrm{Al}_{2} \mathrm{O}_{3}$ ) at $n=10^{12} \mathrm{~cm}^{-2}$ over the range of $T=100$ to $600 \mathrm{~K}$ for $d=0.3 \mathrm{~nm}$. The upper and lower bounds in each panel correspond to $G_{\mathrm{el}}$ for $d=0$ and $d=0.6 \mathrm{~nm}$.

tunability should also be observable for the commonly used $\mathrm{SiO}_{2}$-supported TMD field-effect transistors because classical MD simulation results for the TBC of the $\mathrm{MoS}_{2}-\mathrm{SiO}_{2}$ interface (15.6 MW $/ \mathrm{K} / \mathrm{m}^{2}$ in Ref. [21], 12.2-23.5 MW $/ \mathrm{K} / \mathrm{m}^{2}$ in Ref. [31], and $25.6 \pm 3.3 \mathrm{MW} / \mathrm{K} / \mathrm{m}^{2}$ in Ref. [6]) suggest that $G_{\mathrm{ph}}$ should be in the 10 to $25 \mathrm{MW} / \mathrm{K} / \mathrm{m}^{2}$ range at room temperature for the $\mathrm{TMD}-\mathrm{SiO}_{2}$ interface, comparable to the change in $G_{\mathrm{el}}$ from 0 to $39 \mathrm{MW} / \mathrm{K} / \mathrm{m}^{2}$ when $n$ increases from 0 to $10^{12} \mathrm{~cm}^{-2}$. The tunability of $G_{\mathrm{el}}$ also suggests that the gate voltage can be adjusted to modulate cross-plane heat transfer between the TMD and the substrate. One can exploit this effect to create gate-tunable thermal insulators by layering the TMD with other 2D materials [32] and then using a gate metal electrode to either raise or lower the TBC to facilitate or inhibit heat transfer at the TMD-substrate interface.

Figures 3(a) to 3(f) show the temperature dependence of $G_{\mathrm{el}}$ at $n=10^{12} \mathrm{~cm}^{-2}$ for $d=0.3 \mathrm{~nm}$ from $T=100$ to $600 \mathrm{~K}$, a temperature range that is experimentally accessible. We find that $G_{\mathrm{el}}$ increases monotonically with $T$ in this temperature range for the TMD-SiO 2 and TMD- $\mathrm{Al}_{2} \mathrm{O}_{3}$ interfaces because the phonon population increases with $T$. On the other hand, $G_{\text {el }}$ for the TMD-HfO ${ }_{2}$ interface exhibits a similar trend (i.e., $d G_{\mathrm{el}} / d T>0$ ) initially, but plateaus and then starts to decrease with the temperature at higher $T$ because the expression in Eq. (10) scales as $\sim N_{\gamma}^{\mathrm{Eq}} / T^{2} \propto 1 / T$ at higher $T$ (i.e., $\left.k_{B} T \gg \hbar \omega_{\mathrm{SO} 1}\right)$ given the relatively small $\mathrm{SO} 1$ phonon frequency $\left(\omega_{\mathrm{SO} 1}=21.26 \mathrm{meV}\right)$ in $\mathrm{HfO}_{2}$. This difference in the behavior of the temperature dependence of $G_{\mathrm{el}}$ for different substrates can be used to distinguish the effects of the electronic TBC. With $\mathrm{Al}_{2} \mathrm{O}_{3}$ as the substrate, we predict that the TBC contribution from $G_{\mathrm{el}}$ is not only large (relative to the other substrates), but is expected to have a pronounced temperature dependence that can be verified in experiments, while with $\mathrm{HfO}_{2}$, the temperature dependence is predicted to be significantly weaker. The distinct temperature dependence of $\mathrm{HfO}_{2}$ and $\mathrm{Al}_{2} \mathrm{O}_{3}$ suggests that different substrate materials can be used for heat-transfer applications at different temperature regimes. $\mathrm{HfO}_{2}$ can be used at low temperatures $(T<200 \mathrm{~K})$, while $\mathrm{Al}_{2} \mathrm{O}_{3}$ can be used at higher temperatures $(T>300 \mathrm{~K})$. 


\section{SUMMARY AND CONCLUSION}

In this work, we have developed a theoretical model of electronic thermal boundary conductance (TBC) via the remote phonon scattering of electrons in single-layer $\mathrm{MoS}_{2}$ and $\mathrm{WS}_{2}$ supported on dielectric substrates. We have verified that screened electron-phonon interactions are necessary for realistic predictions. Our model predicts that the electronic TBC is highly dependent on the electron density and is strongly tunable using the gate electrode in field-effect transistors, with the strongest effect seen for $\mathrm{Al}_{2} \mathrm{O}_{3}$. We have also identified the temperature dependence of the electronic $\mathrm{TBC}$ for each dielectric substrate and the temperature regimes at which each substrate material is more effective in interfacial heat transfer. This strong dependence of the electronic TBC on the electron density can be exploited for the design of novel thermal devices with gate voltage-modulated crossplane heat-dissipative properties.

\section{ACKNOWLEDGMENTS}

The authors gratefully acknowledge support from the Science and Engineering Research Council through Grant No. 152-70-00017 and the use of computing resources at the A*STAR Computational Resource Centre and National Supercomputer Centre, Singapore. We also thank Yongqing Cai (University of Macau) for assistance with the DFT calculations.

\section{APPENDIX: ESTIMATION OF TMD-SiO 2 GAP}

The gap size $(d)$ of the TMD- $\mathrm{SiO}_{2}$ interface can be estimated from the distance between the highest substrate atom and the lowest TMD atom. The $\mathrm{WS}_{2}-\mathrm{SiO}_{2}$ and $\mathrm{MoS}_{2}-\mathrm{SiO}_{2}$ heterostructures are optimized structurally within the framework of density functional theory (DFT) using the software package VASP [33]. The DFT-D2 method is adopted to simulate the van der Waals interactions across the interface, while the Perdew-Burke-Ernzerhof functional is used as the exchange-correlation functional together with a cutoff energy of $400 \mathrm{eV}$. The slab models of the heterostructures are constructed with a vacuum layer thicker than $10 \AA$. For the $\mathrm{TMD}-\mathrm{SiO}_{2}$ interface, the heterostructures are constructed from a $3 \times 3$ supercell for the TMD $\left(\mathrm{MoS}_{2}\right.$ or $\left.\mathrm{WS}_{2}\right)$ and a

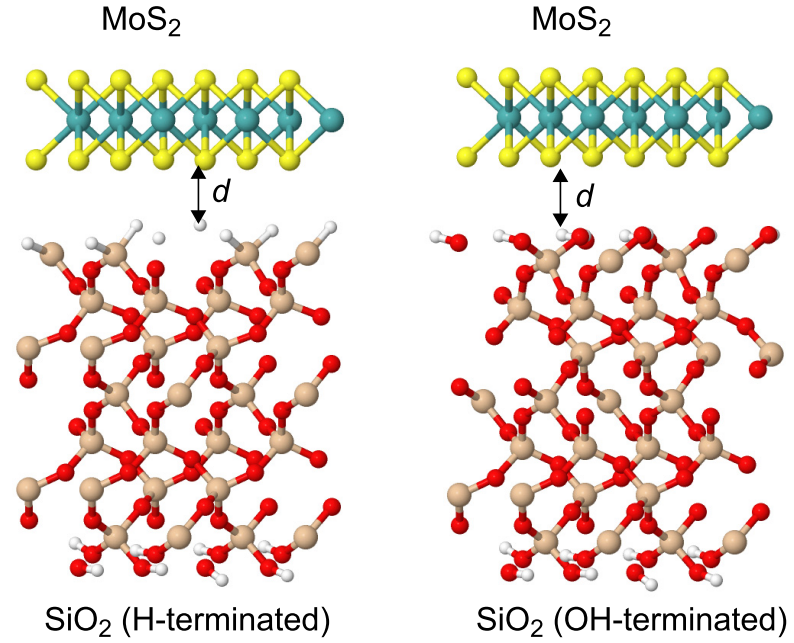

FIG. 4. Side view of the $\mathrm{MoS}_{2}-\mathrm{SiO}_{2}$ heterostructures used in our density functional theory (DFT) calculations to estimate the gap size $d$. We obtain $d=0.253 \mathrm{~nm}$ (H-terminated $\mathrm{SiO}_{2}$ surface on the left) and $0.306 \mathrm{~nm}\left(\mathrm{OH}\right.$-terminated $\mathrm{SiO}_{2}$ surface on the right). The Mo, $\mathrm{S}, \mathrm{H}, \mathrm{Si}$, and $\mathrm{O}$ atoms are colored in blue, yellow, white, beige, and red, respectively. The images are generated using JMOL [34].

$2 \times 2$ supercell for the $\mathrm{SiO}_{2}(001)$ surface to ensure a better lattice match with lattice strains smaller than $2 \%$. To simulate the $\mathrm{SiO}_{2}$ substrate, a slab model with seven $\mathrm{Si}$ layers is used and the atoms in the bottom $\mathrm{O}-\mathrm{Si}-\mathrm{O}$ atomic layers are saturated with hydrogen atoms and fixed during structural optimization. We adopt a $3 \times 3 \times 1$ Monkhorst-Pack (MP) grid for $k$-point sampling for the TMD-SiO $\mathrm{S}_{2}$ heterostructures. All the atomic models are fully relaxed until the forces are smaller than $0.005 \mathrm{eV} / \AA$.

Figure 4 shows the $\mathrm{MoS}_{2}-\mathrm{SiO}_{2}$ heterostructures for different $\mathrm{SiO}_{2}$ surface configurations $(\mathrm{H}$ terminated and $\mathrm{OH}$ terminated), similar to those used in Ref. [8]. We estimate $d=$ 0.253 and $0.306 \mathrm{~nm}$ for the $\mathrm{H}$-terminated and $\mathrm{OH}$-terminated $\mathrm{SiO}_{2}$ surface, respectively. This shows that the estimate of $d$ can vary with the chemical structure of the substrate surface. Hence, it is simpler to approximate $d=0.3 \mathrm{~nm}$ for the $\mathrm{MoS}_{2}-\mathrm{SiO}_{2}$ interface. Our calculations, repeated for the different $\mathrm{WS}_{2}-\mathrm{SiO}_{2}$ interfacial configurations, such as in Fig. 4, also suggest that $d=0.3 \mathrm{~nm}$ for the $\mathrm{WS}_{2}-\mathrm{SiO}_{2}$ interface.
[1] X. Li, L. Yang, M. Si, S. Li, M. Huang, P. Ye, and Y. Wu, Adv. Mater. 27, 1547 (2015).

[2] D. Lembke, S. Bertolazzi, and A. Kis, Acc. Chem. Res. 48, 100 (2015).

[3] E. Pop, Nano Res. 3, 147 (2010).

[4] Z.-Y. Ong and M.-H. Bae, 2D Mater. 6, 032005 (2019).

[5] Z.-Y. Ong, B. Qiu, S. Xu, X. Ruan, and E. Pop, J. Appl. Phys. 123, 115107 (2018).

[6] S. V. Suryavanshi, A. J. Gabourie, A. Barati Farimani, and E. Pop, J. Appl. Phys. 126, 055107 (2019).

[7] B. N. J. Persson, A. I. Volokitin, and H. Ueba, J. Phys.: Condens. Matter 23, 045009 (2011).

[8] Z.-Y. Ong, Y. Cai, and G. Zhang, Phys. Rev. B 94, 165427 (2016).
[9] Z.-Y. Ong, Phys. Rev. B 95, 155309 (2017).

[10] G. C. Correa, C. J. Foss, and Z. Aksamija, Nanotechnology 28, 135402 (2017).

[11] K. Hess and P. Vogl, Solid State Commun. 30, 797 (1979).

[12] M. V. Fischetti, D. A. Neumayer, and E. A. Cartier, J. Appl. Phys. 90, 4587 (2001).

[13] A. Konar, T. Fang, and D. Jena, Phys. Rev. B 82, 115452 (2010).

[14] K. Zou, X. Hong, D. Keefer, and J. Zhu, Phys. Rev. Lett. 105, 126601 (2010).

[15] Z. Yu, Z.-Y. Ong, Y. Pan, Y. Cui, R. Xin, Y. Shi, B. Wang, Y. Wu, T. Chen, Y.-W. Zhang, G. Zhang, and X. Wang, Adv. Mater. 28, 547 (2016).

[16] Z.-Y. Ong, M. V. Fischetti, A. Y. Serov, and E. Pop, Phys. Rev. B 87, 195404 (2013). 
[17] Z. Yu, Y. Pan, Y. Shen, Z. Wang, Z. Y. Ong, T. Xu, R. Xin, L. Pan, B. Wang, L. Sun, J. Wang, G. Zhang, Y. W. Zhang, Y. Shi, and X. Wang, Nat. Commun. 5, 5290 (2014).

[18] A. Taube, J. Judek, A. Lapinska, and M. Zdrojek, ACS Appl. Mater. Interfaces 7, 5061 (2015).

[19] E. Yalon, C. J. McClellan, K. K. H. Smithe, M. Munoz Rojo, R. L. Xu, S. V. Suryavanshi, A. J. Gabourie, C. M. Neumann, F. Xiong, A. B. Farimani, and E. Pop, Nano Lett. 17, 3429 (2017).

[20] E. Yalon, O. B. Aslan, K. K. H. Smithe, C. J. McClellan, S. V. Suryavanshi, F. Xiong, A. Sood, C. M. Neumann, X. Xu, K. E. Goodson, T. F. Heinz, and E. Pop, ACS Appl. Mater. Interfaces 9, 43013 (2017)

[21] P. Yasaei, C. J. Foss, K. Karis, A. Behranginia, A. I. ElGhandour, A. Fathizadeh, J. Olivares, A. K. Majee, C. D. Foster, F. Khalili-Araghi, Z. Aksamija, and A. Salehi-Khojin, Adv. Mater. Interfaces 4, 1700334 (2017).

[22] Y. K. Koh, A. S. Lyons, M. H. Bae, B. Huang, V. E. Dorgan, D. G. Cahill, and E. Pop, Nano Lett. 16, 6014 (2016).

[23] G. D. Mahan, Many-Particle Physics (Springer-Verlag, Boston, 2000).
[24] P. F. Maldague, Surf. Sci. 73, 296 (1978).

[25] Z.-Y. Ong and M. V. Fischetti, Phys. Rev. B 86, 121409(R) (2012).

[26] Z. Jin, X. Li, J. T. Mullen, and K. W. Kim, Phys. Rev. B 90, 045422 (2014).

[27] Z.-Y. Ong and M. V. Fischetti, Phys. Rev. B 86, 165422 (2012).

[28] S. V. Rotkin, V. Perebeinos, A. G. Petrov, and P. Avouris, Nano Lett. 9, 1850 (2009).

[29] J. H. Davies, The Physics of Low-dimensional Semiconductors, 1st ed. (Cambridge University Press, New York, 1997).

[30] C. J. Foss and Z. Aksamija, 2D Mater. 6, 025019 (2019).

[31] H. Farahani, A. Rajabpour, M. Khanaki, and A. Reyhani, Comput. Mater. Sci. 142, 1 (2018).

[32] S. Vaziri, E. Yalon, M. M. Rojo, S. V. Suryavanshi, H. Zhang, C. J. McClellan, C. S. Bailey, K. K. Smithe, A. J. Gabourie, V. Chen, S. Deshmukh, L. Bendersky, A. V. Davydov, and E. Pop, Sci. Adv. 5, eaax1325 (2019).

[33] G. Kresse and J. Furthmüller, Phys. Rev. B 54, 11169 (1996).

[34] Created by Jmol: An open-source JAVA viewer for chemical structures in 3D (http://www.jmol.org/). 